Copyright $@ 2010$ Institute of Electrical and electronics Engineers, Inc.

All Rights reserved.

Personal use of this material, including one hard copy reproduction, is permitted.

Permission to reprint, republish and/or distribute this material in whole or in part for any other purposes must be obtained from the IEEE.

For information on obtaining permission, send an e-mail message to stds-igr@ieee.org.

By choosing to view this document, you agree to all provisions of the copyright laws protecting it.

Individual documents posted on this site may carry slightly different copyright restrictions.

For specific document information, check the copyright notice at the beginning of each document. 


\section{If Cross-functional Teams are the Answer, What is the Question?}

\author{
W K Daniel Pun \\ School of Computing Sciences \\ CQUniversity Australia \\ Rockhampton QLD 4702 Australia \\ D.Pun@cqu.edu.au
}

\author{
Ricardo Santa \\ School of Law and Business \\ Charles Darwin University \\ Darwin NT 0909 Australia \\ Ricardo.Santa@cdu.edu.au
}

\begin{abstract}
This study analyses how cross-functional teams' impact on value-relevance is expected to build and maintain good quality intelligent information systems, and also on operational performance after the implementation of intelligent information systems in firms. The phenomenon of cross-functional teams and the structural relationships were evaluated by using multiple regression analysis. Operational effectiveness and system effectiveness are also considered in the improvement of operational performance. The current results indicate that the quality of information, and the quality and speed provided from operational effectiveness, and the quality of the services supplied from systems effectiveness are the key elements for cross-functional teams to improve operational performance and maintain a good quality of intelligent information system.
\end{abstract}

Keywords-Cross-functional Teams, Intelligent Information Systems

\section{INTRODUCTION}

Most of firms involved in the implementation process of technological innovation are increasingly using crossfunctional teams to enhance their competitiveness [3], and responsiveness to market changes [1]. Despite the recognition of the importance of such factors, the extent to which these cross-functional project teams help firms to increase the effectiveness of the operations, and to continuously improve after the implementation of technological innovations, has received little attention from researchers [4]. For instance, Harrison and Storey [5] and Ahmad and Schroeder [6] conducted studies to link human resources issues, such as crossfunctional teams, with operations management, and argued that operations management literature tends to either ignore, or pay little attention to, human resources issues, even given the critical role of people in achieving superior performance in the operations of service firms. Given the limited empirical studies that specifically investigated cross-functional teams and their impact on the alignment between technological innovation and operational performance in service firms, this research uses a quantitative approach to investigate the stated issue. This research has been trying to address the question "Who plays an important role in both the effectiveness of the operations and the effectiveness of the implemented technological innovatives such as intelligent information systems?" This research analyses the results of a survey of employees in firms from public and private sectors, and aims to build on the existing literature and refine the theoretical framework.

\section{A. Cross-Functional Teams}

In the implementation process of technological innovations some firms are increasingly using human resource factors such as cross-functional teams [2], because cross-functional teams are often understood as the cooperation or collaboration of individuals drawn from various functional areas [3]. This crossfunctional cooperation brings ideas, knowledge, expertise and innovation to the project. For instance, Tidd, Bessant \& Pavitt [1] stated that cross-functional teams have more to offer than individuals in terms of idea generation, flexibility to solutions developed, and a mechanism for bridging boundaries to create innovative solutions within firms. Likewise, a company with a strong culture of innovation leverages relationships across boundaries and through the extended enterprise, and encourages people to reach further and more faster to gain or spread knowledge [7] [8]. Cross-functional teams provide a substantial range of ideas, learning and improvements that can be applied to the firms [9]. Thus, firms need to use crossfunctional teams in the implementation of innovative technologies, such as intelligent information systems, with the aim of bringing ideas, learning, knowledge, expertise and innovation into the project. Managers' efforts to create complex networks to connect their people and partners globally, and to empower employees for cross-functional teams to operate efficiently, however, could be seen as challenging if key factors to succeed are not appropriately defined.

How to obtain successful cross-functional teams have become very important. Many factors, mentioned by [10], such as empowering project teams, establishing project climate, the human resources of the teams, and the establishment of goals, have been commented in the literature by different researchers when looking at how to achieve successful crossfunctional teams. The establishment of clear and consistent goals provides the members of the cross-functional team with a common boundary or frame of reference, and keeps the teams oriented towards common tasks and outcomes. This establishment of clear and consistent goals in turn promotes a higher level of cross-functional cooperation [3]. In addition, the team leader also may play an enabling role in fostering cooperation among team members, to keep the team focus 
on its goals [10]. Successful use of cross-functional teams in the implementation of technological innovations requires the establishment of clear and consistent goals. Additionally, it is important to identify the expectation of stakeholders as they come from different functional areas and diverse cultures, as this diversity could bring miscommunication or misunderstandings to the cross-functional team.

In 1997, Pitta and Franzak [25] suggested that firms involving external and internal cross-functional members create a boundary spanning team. Boundary spanning is tied closely with the management of technology, innovation, and strategic implementation to achieve organisational objectives. As stated by Schein [12] in 1996, actors such as managers, engineers and operators-users belong to different cultures, making it difficult to communicate and interact with each other. Boundary spanning agents are viewed as communication stars Tushman and Scanlan [26] and can effectively communicate widely within their work unit, across work units and outside their firm. Kel$\log$ et al. [?] argued that boundary spanning agents are able to act as translators, brokers or mediators. These boundary spanning actors or conciliators of cross-functional teams would provide the essential communication link between members of diverse work groups, helping in the interpretation of messages and reducing the barriers to communication, such as, selective perception of what one hears or sees based on their own needs (Robbins et al. 2008). Cross-functional teams can reduce misunderstanding that arises in the different values found within functional areas, if the cross-functional team members are able to develop a shared language and shared mental models. By doing this, actors from different functional areas can share information at all stages of the implementation of technological innovations, making it easier to identify problem areas early in the process and finding solutions that are shared by the team members in the same language.

\section{B. Operational Effectiveness}

For changing market conditions, service firms need learning processes to build the flexible capability to reconfigure and transform their processes. An increasing number of factors are prompting firms to seek ways to operate more efficiently and to ensure they have effective operational processes [13] [14]. This involves the need to deliver value-adding products or services of exceptional quality, on time and at a competitive price. Thus, firms attempting to meet these objectives need to pay attention to their operational effectiveness, as this is a primary driver of business performance [14]. Operational effectiveness refers to the ability to establish processes, based on core capabilities within the firms which work well [15]. Operational effectiveness involves improving process performance by leading and controlling the processes within the firm, as well as measuring and improving the processes. A better use of resources through these core processes enables the firms to eliminate waste, reduce costs, adapt more appropriate technological innovation and therefore perform better than competitors [15].

The five performance dimensions or objectives a firm seeks to fulfil to attain operational effectiveness are cost, quality, reliability, flexibility and speed [16] [13]. Creating competitive advantage and improving operational performance is not an overnight task. Therefore, excelling on some of the objectives gives and being competitive on each of the others give a firm an edge in the market [28]. Improving cost performance means that a firm seeks the elimination of waste which comes from efficiencies attained in processes such as purchasing, production and staff performance [29]. An appropriate disaggregation of the cost components impacting on the total cost performance of a firm gives the opportunity to identify the areas for improvement [14]. Improving on quality provides firms with the opportunity to bridge the gap between what they are capable of offering and what customer demands. Quality is viewed as a consistent provision of products and services that satisfy customers, rather than only minimizing defects and conforming specifications without any clear market orientated continuous improvement [29]. The third operational performance objective concerns being flexible, which includes a firms ability and the extent to adjust (what it does, how it does and when it does) to changes in response to customers requirements [30]. Improving on speed prompts a firm to be able to shorten the time between the service request and delivery of the service, with the frequency and at times requested by customers [13]. Improving in time reflects a growing pressure on firms not just to introduce new products or services but to do it faster than competitors [31]. At the same instance, new product or service development is an important capability as the environment is constantly changing [31]. Reliability means that firms processes consistently perform as expected over time. That is, customers are satisfied by firms that provide services as agreed and do not fail over a period of time [17].

\section{System Effectiveness}

System effectiveness has been described as the extent to which intelligent information systems contribute to achieving firms goals and benefits [18]. The initial DeLone and McLeans [19] model contained five variables: system quality, information quality, perceived usefulness, user satisfaction, and intelligent system use. In 2003 DeLone and McLean [18] revised their model to include six interrelated dimensions of intelligent information systems success, to measure the dependent variable intelligent system effectiveness. The following list describes the six dimensions described in the DeLone and McLean [18] 10 years update success model:

1) System quality measures technical efficacy the desired characteristics of the system. This assessment is based on the performance and productivity of the system [18] [32];

2) Information quality is the measurement of output from IIS. It measures semantic success characteristics of the information and its desired form; the degree to which information produced has the attributes of content, accuracy, and format required by the user [18] [32]; 
3) Service quality is the level of service received by the users of IIS and the manner in which the service is provided by the information systems department as it influences the degree of satisfaction with an intelligent information system [33] [18];

4) Use and user satisfaction measure effectiveness success through studies that attempt to analyse and measure the interaction of the information product with its recipients, the degree to which the user believes that using a particular system has enhanced his or her job performance. User satisfaction is defined as the users response to the use of the output of an intelligent information system, the psychological state of the user after using the intelligent information system;

5) Individual impact is the effect the information has on the behaviour of the user, including improving personal or departmental performance, relating to what influences the information product has on management decisions. This impact occurs when the information is received and understood by the users, and applied to their jobs; and

6) Organisational impact derives from research that investigated the effect of the information product on organisational performance [34] [18] [32].

Even if the DeLone and McLean models [19] [18] presented an integrated view of the concept of information system success in a more comprehensive taxonomy [35], there is still a general concern that has been addressed by Lang [35] on how to more effectively deal with the issue of information system success measurement. Thus, it is increasingly expected that IIS effectiveness should be measured in terms of the real operational benefits rather than through the achievement of information system outcomes only. Furthermore, It is important to understand key elements of the relationship between system effectiveness and operational effectiveness and to have a real picture of the impact of technological innovation implementation in the improvements on operational performance.

The main purpose of this research is to build on and extend the existing literature and to put forward a theoretical framework that examines the following propositions:

1) That there is a relationship between cross-functional teams, system effectiveness, operational effectiveness and continuous improvement of operational performance.

2) That a limited number of factors have the potential to explain the alignment between system effectiveness and operational effectiveness.

3) That cross-functional project teams influence the continuous improvement of the operational performance.

\section{RESEARCH Issues AND Methodology}

This research is undertaken with an exploratory purpose, as the alignment between system effectiveness, operational effectiveness, and cross-functional teams and their influence in the continuous improvement of operational performance in service firms, has had little academic investigation. According to Sekaran [20] exploratory study is undertaken when there is a lack of understanding of the problem, which leads to an unstructured problem design. Quantitative data was gathered through a self administered questionnaire. The questionnaire was administered to managers, engineers (technologist), and administrative and operational staff as, according to Orlikowski and Gash [11], different actors in a firm have different assumptions, expectations, knowledge and perceptions of technological innovation. The questionnaire was also administered in firms from the electricity distribution and retail sector and from the higher education sector, all of which had recently implemented an intelligent system.

In the process of constructing measures of key variables and refining the survey instrument, four pilot tests were conducted. These pilot tests enabled the introduction of a number of revisions carried out to improve the survey instrument between the initial draft and the final instrument.

\section{RESULTS}

\section{A. Assessment of Data Validity}

The questionnaires were emailed to employees such as managerial or executive roles in information technology and intelligent information systems, engineering roles, operators, and general staff in the selected service firms. Of the 450 surveys distributed among the service firms from the electricity distribution, retail sector and higher education sector, 144 were returned ( $32 \%$ response). Each returned questionnaire was reviewed for completeness and, of the 144, 6 were considered unusable due to large amounts of missing data, lack of involvement of the respondent in the use of intelligent system, or the impossibility of identifying the role of the respondent (manager, engineer or operator-user).

The cross-functional teams section of the questionnaire reported a reasonably high Cronbach's Alpha coefficient of 0.743. The system effectiveness section of the questionnaire reported a Cronbach's Alpha coefficient of 0.859. The operational effectiveness section reported a Cronbachs Alpha coefficient of 0.936. This high coefficient supported the argument for bringing the dimensions of operational effectiveness into the system effectiveness context to have a more comprehensive understanding of the real effectiveness of the intelligent system implementation. The continuous improvement of operational performance section reported a Cronbach's Alpha coefficient of 0.862. These Cronbachs Alpha coefficients indicated a high level of internal consistency within these measures as the generally accepted lower limit is 0.7 , though some studies allow 0.6; for example, Hair et al. [21]. The strength and nature of relationships between the dimensions stemming from system effectiveness and the performance objectives stemming from operational effectiveness and cross-functional teams were investigated using the Pearson product-moment correlation coefficient. The high correlation and significance means that the performance objectives stemming from operational effectiveness can be used to measure the impact of the implementation of technological innovations such as intelligent information systems on operational effectiveness, and the important impact of cross-functional teams in the alignment between system 


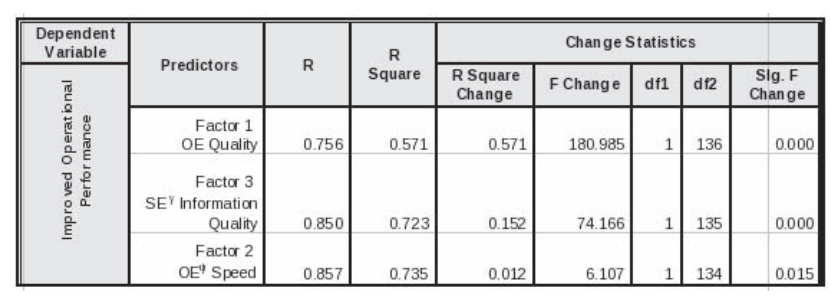

Fig. 1. Predictors of improved operational performance

effectiveness and operational effectiveness. Furthermore, the dimensions stemming from operational effectives produce a more comprehensive model than the traditional systems effectiveness success model developed by DeLone and McLean [18].

\section{B. Factor Analysis}

As the main purpose of the study was to explore the alignment between system effectiveness and operational effectiveness and the role played by cross-functional teams in the stated alignment and in the continuous improvement of operational performance, the next step in the data analysis is to perform a factor analysis to reduce the dimensionality and to identify the most important clusters from system effectiveness and operational effectiveness, while at the same time eliminating the items that are less representative. The factor analysis was performed using the maximum likelihood extraction method and oblique rotation method which allows the factors to be correlated. During the factor analysis process, four factors emerged. Hair et al [21] argue that in order to ensure a power level of $80 \%$, a factor loading of 0.55 is significant if the sample size is at least 100 observations at a significant level $(\alpha)$ of 0.05 . Thus, only factor loadings of at least 0.60 have been considered. The solution resulted in a Kaizer-MeyerOlkin (KMO) value of 0.832 with four factors accounting for $52.65 \%$ of the cumulative variance, indicating a satisfactory solution. The four factors are: quality and speed stemming from operational effectiveness, and quality of information and quality of the service stemming from system effectiveness. These four factors also demonstrate the focus that firms need to consider when aligning technological innovations with operational effectiveness. It is expected also that this alignment will assist firms to enhance continuous improvement.

\section{Path Analysis}

Forward multiple regression was used to assess the ability of the two dimensions stemming from system effectiveness, the two performance objectives stemming from operational effectiveness, and cross-functional teams to predict continuous improvement of operational performance. Furthermore, path analysis has assisted in describing the entire structure of linkages between independent and dependent variables [22]. Preliminary analysis was conducted to ensure no violation of the assumptions of normality, linearity, muticollinearity and homoscedascity [23] [24].

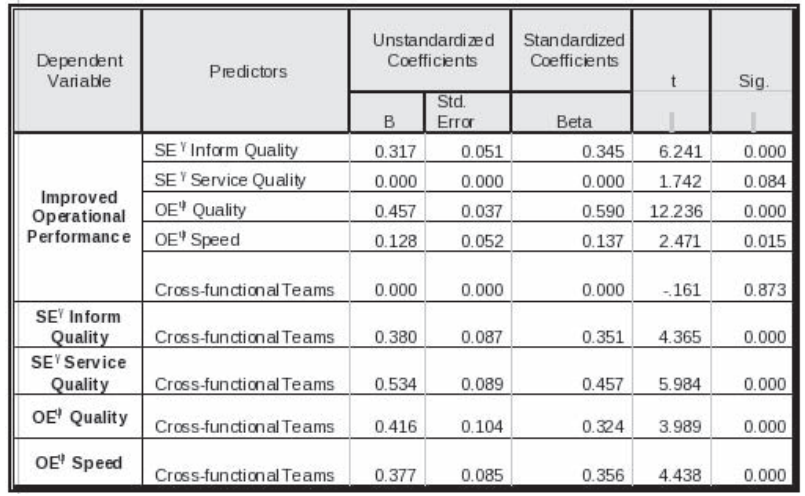

Fig. 2. Path analysis for improved operational performance (IOP)

Fig. 1 represents entry of the set of predictors of Continuous Improvement of Operational Performance. The results show that Factor-1 'Operational Effectiveness Quality' accounted for $57.1 \%$ of the variance ( $\mathrm{R}$ square) in the success of Continuous Improvement of Operational Performance. Fig. 1 also shows that Factor-3 'System Effectiveness Information Quality' accounted for $15.2 \%$ of the variance ( $\mathrm{R}$ square Change). Entry level of the independent variable Factor-2 'Operational Effectiveness Speed', resulted in a significant $F$ change, Improvement of Operational Performance, and increased variance explained by $1.2 \%$ (R Square Change). The model as a whole explains $73.5 \%$ ( $\mathrm{R}$ square) of the Continuous Improvement of Operational Performance. Factor4 'System Effectiveness Service Quality' and Cross-functional Teams have been deleted as the variance accounted ( $\mathrm{R}$ square) less than $1.2 \%$ and a significance $p>.05$, and are not seen as predictors of Continuous Improvement of Operational Performance.

The path coefficients between Continuous Improved of Operational Performance and the two dimensions stemming from system effectiveness, the two performance objectives stemming from operational effectiveness and Cross-functional Teams were obtained by regressing the former on the latter [22]. The results from the coefficients table (see Fig. ??) generated from the regression analysis show that only three dimensions or performance objectives entered our prediction equation (Factor 1 Operational Effectiveness Quality: $\beta=.59$; Factor 3 System Effectiveness Information Quality: $\beta=.35$; Factor 2 Operational Effectiveness Speed: $\beta=.14$ ).

Quality of the service stemming from system effectiveness and Cross-functional Teams has no predictive power or contribution on Continuous Improvement of Operational Performance. Moreover, the results from the coefficients table (Fig. ??) show that Cross-functional Teams is a significant predictor of the two dimensions stemming from system effectiveness and the two performance objectives stemming from operational effectiveness (Factor 1 Operational Effectiveness Quality: $\beta=.32$; Factor 2 Operational Effectiveness Speed: $\beta=.36$; Factor 3 System Effectiveness Information Quality: $\beta=.35$; Factor 4 System Effectiveness Service Quality: 
$\beta=.46)$.

\section{CONCLUSION}

In answering the research question 'Who plays an important role in both the effectiveness of the operations and the effectiveness of the implemented technological innovatives such as intelligent information systems?' this study has demonstrated that the cross-functional teams have an important role in both the effectiveness of the operations and the effectiveness of the implemented innovative technology. Consequently, operational performance can be improved by cross-functional teams, but only via the effectiveness of operations and the effectiveness of the technological innovation.

The findings from this study suggest that there is a strong relationship between cross-functional teams and operational effectiveness, as well as between operational effectiveness and continuous improvement of operational performance. This indicates that when formulating strategies it is not only important to put an emphasis on system effectiveness but also on the incorporation of operational performance objectives in order to maximise the continuous improvement of operational performance. Therefore, the implementation of technological innovations such as intelligent information systems should be focused on attaining the needs of the operations in the performance objectives: cost, quality, reliability, flexibility, and speed. Special attention, however, must be given to the performance objectives' quality and speed, as this study has demonstrated that these two factors are the most important in achieving operational effectiveness. This research has also shown that the goals for cross-functional teams to operate efficiently are: quality of the information, quality of the service and quality and speed of the operations. This research supports McDonoughs [10] conclusion, that the use of cross-functional teams is significantly associated with performance, indicating that employing cross-functional teams does have a positive impact on project performance.

\section{REFERENCES}

[1] J. Tidd, J. Bessant, J, K. and Pavitt, K, Managing Innovation, USA: John Wiley and Sons, Ltd, 2001.

[2] B. Daily and S. Huang, "Achieving sustainability through attention to human resource factors in environmental management", International Journal of Operations \& Production Management, vol. 21, no. 12, pp. 1539-1552, 2001.

[3] M. Pinto, J. Pinto, and J. Prescott, "Antecedents and consequences of project team cross-functional cooperation", Management Science, vol. 39, no. 10 , pp. 1281-1297, 1993.

[4] M. Koulikoff-Souviron and A. Harrison, "The pervasive human resource picture in interdependent supply relationships", International Journal of Operations \& Production Management, vol. 27, no. 1, pp. 8-27, 2007.

[5] A. Harrison and J. Storey, "New wave manufacturing strategies: operational, organisational and human dimensions", International Journal of Operations \& Production Management, vol. 16, no. 2, pp. 63-76, 1996.

[6] S. Ahmad and R. Schroeder, "The impact of human resource management practices on operational performance: recognising country and industry differences", Journal of Operations Management, vol. 21, pp. 19-43, 2003.

[7] R. Moss, "A Culture of Innovation”, Executive Excellence, vol. 17, no. 8, pp. 10-11, 2000.

[8] S. Robbins, Essentials of Organisational Behaviour, Upper Saddle River: San Diego State University, 1997.
[9] H. Boer, S. Caffyn, M. Corso, P. Coughlan, J. Gieskes, M. Magnusson, S. Pavesi, and S. Ronchi, "Knowledge and continuous innovation: the CIMA methodology", International Journal of Operations and Production Management, vol. 21, no. 4, pp. 490-504, 2001.

[10] E. McDonough, "Investigation of factors contributing to the success of cross-functional teams", Journal of Production and Innovation Management, vol. 17, no. 3, pp. 221-235, 2000.

[11] W. Orlikowski and D. Gash, "Technological frames: making sense of information technology in organisations", ACM Transactions on Information Systems, vol. 12, no. 2, pp. 174-207, 1994.

[12] E. Schein, "Three cultures of management: the key to organisational learning", Sloan Management Review, vol. 38, no. 1, pp. 9-20, 1996.

[13] T. Hill, "Strategic Context and Managerial Analysis", Operations Management, Pearson Education Limited, 2000.

[14] N. Slack, S. Chambers, and R. Johnston, Operations Management, 4th edn, Pearson Education Limited, 2004.

[15] M. Porter, "What is strategy?", Harvard Business Review, 1996.

[16] G. White, "A survey and taxonomy of strategy-related performance measures for manufacturing", International Journal of Operations and Production Management, vol. 16, no. 3, pp. 42-61, 1996.

[17] L. M. Corbett, "Delivery windows - a new view on improving manufacturing flexibility and on-time delivery performance", Production and Inventory Management Journal, vol. 33, no. 3, pp. 74-79, 1992.

[18] W. DeLone and E. McLean, "The DeLone and McLean model of information system success: a ten-year update", Journal of Management Information Systems, vol. 19, no. 4, pp. 9-30, 2003.

[19] W. DeLone and E. McLean, "Information systems success: the quest for the dependent variable", Information Systems Research, vol. 3, no. 1, pp. 60-95, 1992.

[20] U. Sekaran, Research Methods for Business, 4th edn, John Wiley and Sons, 2003.

[21] J. F. Hair, R. E. Anderson, R. L. Tatham, and W. C. Black, Multivariate Data Analysis, New Jersey, 1998.

[22] R. Ho, Handbook of Univariate and Multivariate Data Analysis and Interpretation with SPSS, Chapman \& HAll CRC, 2006.

[23] R. Cooksey, Illustrating Statistical Procedures for Business, Behavioural \& Social Science Research, Tilde University Press, 2007.

[24] J. Pallant, SPSS Survival Manual, Crows Nest, NSW, Australia: Allen \& Unwin, 2007

[25] D. Pitta, \& F. Franzak, "Boundary spanning product development in consumer markets: learning organisation insights", Journal of Product and Brand Management, vol. 6, no. 4, pp. 235-249, 1997.

[26] M. Tushman \& T. Scanlan, "Characteristics and external orientations of boundary spanning individuals", Academy of Management Journal, vol. 24, no. 1 , pp. 83-98, 1981.

[27] K. Kellogg, W. Orlikowski, \& J. Yates, "Life in the trading zone: structuring coordination across boundaries in postbureaucratic organisations", Organization Science, vol. 17, no. 1, pp. 22-44, 2006

[28] S. C. Wheelwright \& H. Bowen, "The Challenge of manufacturing advantage", Production and Operations Management, vol. 5, no. 1, pp. 59-77, 1996.

[29] R. S. Russell \& B. W. Taylor, Production and operations management: focusing on quality and competitiveness, Prentice hall, 1995.

[30] Slack, "The manufacturing advantage", Mercury Books, London, 1991.

[31] J. Tidd, J. Bessant, \& K. Pavitt, "Managing Innovation", John Wiley \& Sons, Ltd., 2001.

[32] J. Nielsen, "Critical success factors for implementing ERP system", in Qualitative Case Studies on Implementation of Enterprise Wide Systems, Idea Group Inc., pp. 211-231, 2005.

[33] L. F. Pitt, R. T. Watson, \& C. V. Kavan, "Service quality: a measure of information systems effectiveness", MIS Quarterly, vol. 19, no. 2, pp. 173-188, 1995.

[34] A. Rai, S. Lang, \& R. Welker, "Assessing the validity of IS success models: an empirical test and theoretical analysis", Information System Research, vol. 13, no. 1, pp. 50-69, 2002.

[35] S. Lang, "An empirical examination of the practical importance of selected information systems success measures", School of Accountancy, Southern Illinois University at Carbondale, 2001. 